\title{
Amphipathic helical ordering of the flagellar secretion signal of Salmonella flagellin
}

$$
\text { Orsolya Tőke }{ }^{1} \text { and Ferenc Vondervisztt }{ }^{2,3, *}
$$

${ }^{1}$ Institute of Organic Chemistry, Research Centre for Natural Sciences, Hungarian Academy of Sciences, Magyar tudósok krt. 2, H-1117 Budapest, Hungary

${ }^{2}$ Bio-Nanosystems Laboratory, Research Institute for Chemical and Process Engineering, University of Pannonia, Egyetem u. 10, H-8200 Veszprém, Hungary

${ }^{3}$ Institute of Technical Physics and Materials Science, Centre for Energy Research, Konkoly-Thege u. 29-33, H-1121 Budapest, Hungary

\begin{abstract}
Export of external flagellar proteins requires a signal located within their $\mathrm{N}$-terminal disordered part, however, these regions do not share any significant sequence similarity suggesting that the secondary/tertiary structure might be important for recognition by the export gate. NMR experiments were performed to reveal the conformational properties of the flagellin signal sequence in vitro. It assumed a largely disordered fluctuating structure in aqueous environment, but acquired a folded structure containing an amphipathic helical portion in $50 \% \mathrm{MeOH}$ or upon addition of SDS micelles which are known to promote hydrophobic interactions. Our observations raise the possibility that the signal sequence may partially undergo amphipathic helical ordering upon interaction with the recognition unit of the flagellar export machinery in a similar way as revealed for protein import into intracellular eukaryotic organelles mediated by targeting signals of high diversity.
\end{abstract}

Keywords: type III secretion; flagellar export; export signal; helical ordering; NMR

Abbreviations: FliC, flagellin; TS, targeting signal

*Corresponding author, email: von007@almos.vein.hu 


\section{Introduction}

The bacterial flagellum is a protein-based rotary nanomachine for locomotion which contains a membrane embedded molecular motor rotating a long helical filament [1]. The helical filament is connected to the basal structure via a short, highly curved segment called the hook. While the hook is a helical assembly of $\sim 130$ copies of FlgE subunits, the filament may comprise tens of thousands of flagellin (FliC) subunits and can grow up to $20 \mu \mathrm{m}$. Other components of the filamentous axial portion of the bacterial flagellum are the five rod proteins (FliE, FlgB, FlgC, FlgF, FlgG) forming the axis of the motor, and the hook associated proteins FlgK, FlgL and FliD. FlgK and FlgL are junction proteins that connect the hook to the filament, while FliD forms a capping structure at the distal end of the flagellar filament, which helps the incorporation of flagellin monomers into the filament at the tip.

Flagellar axial proteins forming the structures lying beyond the cytoplasmic membrane are synthesized in the cell and exported by the flagellum-specific protein export apparatus from the cytoplasm to the site of assembly at the distal end of the growing filament [2]. Thousands of subunits must be translocated through the narrow (20-25 Å wide) central channel of the flagellum in a largely unfolded conformation. The flagellar protein export system is located at the cytoplasmic side of the basal part of the flagellum to distinguish flagellar proteins from other cytoplasmic proteins and to facilitate their transportation. The membrane-associated FlhA-FlhB complex of the export apparatus is thought to be responsible for export substrate recognition. The flagellar export machinery belongs to the family of the type III secretion systems, which also include those for secretion of virulence factors by a wide variety of pathogenic bacteria [3-5]. Recent studies have demonstrated that the proton motive force across the cytoplasmic membrane is responsible for driving the export process $[6,7]$.

In spite of intensive efforts, the nature of the signal directing flagellar protein secretion is still mysterious [8-10]. The protein substrates do not possess a classical cleavable signal sequence or do not share any obvious consensus sequence. Secretion was reported to require a disordered Nterminal secretion signal, but an mRNA encoded signal within the $5^{\prime}$-untranslated region may also 
play a role in facilitating the export process [10]. A growing number of evidence indicates a critical role for the recognition signal located in the N-terminal region of the secreted proteins [11-14]. The identified signal sequences of different flagellar proteins, however, do not show any significant sequence similarity. It is an open question how such dissimilar signals interact with a single export apparatus.

Terminal disorder is a common structural feature of the axial proteins of the bacterial flagellum $[1,15]$. In the case of Salmonella flagellin the disordered terminal regions span the first 66 and the last 44 amino acid residues [16]. The disordered regions of the axial components are highly conserved and predicted to have a potential to fold into amphipathic $\alpha$-helical structures. Signal sequences of the axial proteins lie exclusively within their disordered $\mathrm{N}$-terminal region.

In this work, conformational properties of the export signal of Salmonella flagellin, comprising residues 26-47, were investigated in vitro under various conditions. Although the major portion (residues 32-44) of the signal sequence forms the extended and rather irregular spoke region in the filament core [19], connecting the inner and outer ring structures, our NMR studies demonstrate that it can readily adopt a largely helical conformation in a solvent promoting amphipathic helix formation.

\section{Materials and Methods}

Materials. Deuterated $\mathrm{d}_{25} \mathrm{SDS}$ and $\mathrm{CD}_{3} \mathrm{OH}$ were from Cambridge Isotope Laboratories (Andover, $\mathrm{MA}$, USA). Natural abundance amino acid derivatives were purchased from Novobiochem (Laufelfingen Switzerland) or Reanal (Budapest, Hungary). The 4-methylbenzhydrylamine (MBHA) resin was obtained from Novobiochem (Laufelfingen Switzerland). N, $\mathrm{N}^{\prime}$-diisopropylcarbodiimide (DIC), , 1hidroxybenztriazole (HOBt), 1.8-diazabicyclo[5.4.0]undec-7-ene (DBU), and trifluoroacetic acid (TFA) were products from Fluka (Buchs, Switzerland). Solvents (DMF, DCM, acetonitrile, diethyl-ether) for peptide synthesis and purification were purchased from Reanal (Budapest, Hungary). All other reagents were from Sigma (St. Louis, MO, USA). 
Peptide Synthesis and Purification. The flagellin signal peptide (GTAIERLSSGLRINSAKDDAAG) was synthesized manually by solid phase peptide synthesis on Rink-amide MBHA resin using standard Fmoc/ ${ }^{\mathrm{t}} \mathrm{Bu}$ chemistry. After assembly, the peptide was cleaved from the resin by $10 \mathrm{~mL}$ TFA using 0.75 g phenol, $0.5 \mathrm{~mL}$ distilled water, $0.5 \mathrm{~mL}$ thioanisole and $0.25 \mathrm{~mL}$ ethandithiol as scavengers. Crude products were precipitated by dry diethyl-ether, dissolved in $10 \%$ acetic acid and freeze-dried. The freeze-dried crude product was purified on a semi-preparative Phenomenex Jupiter C18 column (250x10mm I.D.) with $10 \mu \mathrm{m}$ silica (300 ̊̊ pore size) (Torrance, CA, USA). Flow rate was $4 \mathrm{~mL} / \mathrm{min}$. Linear gradient elution was generated using $0.1 \%$ TFA in water as eluent $A$ and $0.1 \%$ TFA in acetonitrile-water $(80: 20, \mathrm{v} / \mathrm{v})$ as eluent B. Peaks were detected at $\lambda=220 \mathrm{~nm}$.

Analytical HPLC was performed on a Knauer (Herbert Knauer GmbH, Berlin, Germany) system using a Phenomenex SYNERGI MAX-RP column (Torrance, CA, USA) as a stationary phase. Linear gradient elution, $0 \mathrm{~min} 0 \% \mathrm{~B} ; 5 \min 0 \%$ B; $50 \mathrm{~min} 90 \%$ B, was used. Flow rate of $1 \mathrm{~mL} / \mathrm{min}$ was applied at ambient temperature. Peaks were detected at $\lambda=220 \mathrm{~nm}$. The samples were dissolved in eluent B. The molecular mass of the peptide was measured by ESI-MS. Positive ion electrospray ionization mass spectrometric analysis was performed on a Bruker Esquire 3000 plus (Germany). The sample were dissolved in acetonitrile-water $(50: 50, \mathrm{v} / \mathrm{v})$, containing $0.1 \%$ acetic acid.

NMR experiments. Aqueous samples with no micelles and samples in methanol were prepared by dissolving $\sim 5 \mathrm{mg}$ of the lyophilized peptide (MW=2202) in either $700 \mu \mathrm{L}$ buffer containing $20 \mathrm{mM}$ potassium-phosphate, $50 \mathrm{mM}$ potassium-chloride, $0.05 \% \mathrm{NaN}_{3}$ and $10 \% \mathrm{D}_{2} \mathrm{O}$ at $\mathrm{pH}=6.3$ or in $700 \mu \mathrm{L}$ $\mathrm{CD}_{3} \mathrm{OH} / \mathrm{H}_{2} \mathrm{O}(1: 1, \mathrm{v} / \mathrm{v})$ mixture. Samples containing SDS micelles were prepared by dissolving $\sim 3 \mathrm{mg}$ peptide in $700 \mu \mathrm{L}$ of $10 \mathrm{mM}$ sodium-phosphate buffer ( $\mathrm{pH}=5.8$ ), containing $200 \mathrm{mM} \mathrm{d}_{25}$-SDS and $10 \%$ $\mathrm{D}_{2} \mathrm{O}$.

One- and two-dimensional solution NMR experiments were carried out on a Varian NMR SYSTEM ${ }^{\text {TM }}$ (600 $\mathrm{MHz}$ for ${ }^{1} \mathrm{H}$ ) four-channel spectrometer using a 5 -mm indirect detection triple resonance $\left({ }^{1} \mathrm{H}^{13} \mathrm{C}^{15} \mathrm{~N}\right.$ ) z-axis gradient probe. Experiments were performed at either $25^{\circ} \mathrm{C}$ (aqueous buffer, $50 \%$ methanol) or $40{ }^{\circ} \mathrm{C}$ (SDS). The ${ }^{1} \mathrm{H}$ resonances were assigned and analyzed as described in [20]. 


\section{Results}

Solution NMR Spectroscopy. The conformation of the signal peptide was investigated in aqueous buffer, in 50\% methanol, and in negatively charged SDS micelles. One-dimensional ${ }^{1} \mathrm{H}$ NMR spectra of the peptide in aqueous buffer $(\mathrm{pH}=6.3)$ show poor chemical shift dispersion (Figure $1 \mathrm{~A}$ ) indicating the lack of a well-defined structure. In the presence of methanol, marked shift changes occur in the amide proton region, demonstrating the adaption of a folded structure (Figure 1B). This is most clearly seen for residues A3, 14, E5, L11, N14, A16. To further mimic the effect of an amphipathic environment on the structure of the export signal, in parallel with the structure determination in methanol, we have also characterized the conformation of the peptide in SDS micelles. Figure 1C shows the amide proton region of the ${ }^{1} \mathrm{H}$ spectrum at a detergent:peptide molar ratio of $\sim 130$. Upon further increasing the relative amount of SDS no further changes in chemical shifts were observed, indicating that under these experimental conditions all of the peptide was bound. Peptide binding is also apparent from the line broadening of resonances as a result of slower tumbling. The chemical shift dispersion of the amide proton region suggests that the micelle-bound peptide must possess some amount of secondary structure.

Resonance Assignment. The assignment of backbone and side chain proton resonances of the signal peptide in all investigated environments was accomplished using the combination of 2D TOCSY, NOESY, and natural abundance ${ }^{13} \mathrm{C}-\mathrm{HSQC}$ experiments. In addition to the assessment of $\mathrm{H}_{\mathrm{N}}-\mathrm{H}_{\text {aliphatic }}$ correlations, the assignment was aided by the $\mathrm{H}_{\mathrm{N}}-\mathrm{H}_{\mathrm{N}}$ region of the NOESY spectra, in which many of the sequential $d_{N N}(i, i+1)$ as well as a few $d_{N N}(i, i+2)$ correlations were detected throughout the peptide backbone. The fingerprint $\mathrm{H}_{\mathrm{N}^{-}} \mathrm{H}_{\alpha}$ region of the peptide obtained in $50 \%$ methanol are shown in Figure 2A. Three residues in the C-terminal half of the peptide chain $(A 16, K 17, D 18)$ exhibit peak doubling indicating the presence of a minor component sensing a different chemical environment in the affected region. For comparison, the corresponding spectral region in SDS is depicted in Figure 2B. In the presence of micelles, to overcome an inefficient relay of magnetization in TOCSY experiments, 2D NOESY spectra acquired with increasing mixing times were included in the analysis 
(data not shown). The full sequence specific assignments of the signal peptide in various environments are given in the Supplementary Information (Tables S1-S3).

NMR Structural Parameters. In aqueous buffer, the $\mathrm{H}_{\alpha}$ chemical shifts of the signal peptide indicate a slight preference for alpha-helical conformation, mostly toward the C-terminal region (I13-D18) of the peptide chain (Figure 3A). However, the lack of long-range distance restraints in NOESY spectra suggests the lack of sufficient intramolecular contacts to form a stable secondary structure. The presence of methanol induces changes in $\mathrm{H}_{\alpha}$ chemical shifts throughout the entire sequence, which are consistent with a more pronounced alpha-helical content. Unlike in the case of methanol, chemical shift changes characteristic of helix-formation induced by SDS micelles are mostly limited to the $\mathrm{N}$-terminal half of the peptide chain (A3-S8). In the C-terminal half, deviations from random coil values appear to be highly similar to that observed in aqueous buffer and show a small helical propensity. Interestingly, the helix-disturbing effect of G10 in the middle of the chain is manifested only in the presence of micelles.

Secondary chemical shifts were also determined for the backbone amide protons. These parameters are known to be related to $\mathrm{H}$-bond lengths [21,22] and have been associated with the location of amino acid residues in $\alpha$-helices [23]. Differences between the observed HN chemical shifts and those expected in a random coil are shown in Figure $3 \mathrm{~B}$ as a function of the amino acid position in methanol and in micelles. While both curves display some extent of periodicity in the Nterminal half with maximum positive (negative) $\Delta \delta_{\mathrm{NH}}$ values at positions I3 (E5) and R6 (S9), toward the middle of the chain the periodicity appears to be lost in both environments. Yet some type of regularity reappears in the C-terminal half between R12 and D19 in both methanol and SDS. Intriguingly, the relative fluctuations along the amino acid sequence are similar in the two environments, only the curves appear to be shifted along the y axis, which is most pronounced in segment S9-S15. Larger in magnitude negative values of $\Delta \delta_{\mathrm{NH}}$ as observed in SDS correspond to longer $\mathrm{NH}----\mathrm{OC}$ distances that is looser helix-stabilizing $\mathrm{H}$-bonds for the micelle-bound peptide. 
In addition to chemical shifts, typical ${ }^{1} \mathrm{H}-{ }^{1} \mathrm{H}$ NOE correlations are indicative measures of peptide secondary structure [24]. While in aqueous buffer, the detected NOESY crosspeaks were mostly limited to intra-residue and sequential $(\mathrm{i}-\mathrm{i}+1){ }^{1} \mathrm{H}-{ }^{1} \mathrm{H}$ correlations, in $50 \% \mathrm{CD}_{3} \mathrm{OH}$ and SDS, several $d_{\alpha N}(i, i+2), d_{\alpha N}(i, i+3), d_{\alpha \beta}(i, i+3)$, and $d_{\alpha \beta}(i, i+4)$ NOE correlations characteristic of alpha-helical conformation have been observed (Figure 3C-D). The $i-i+3, i-i+4$ spatial proximities as well as other long-range NOE-s were particularly prevalent in the N-terminal half in SDS and were detected more uniformly throughout the sequence in methanol. The two distinct segments corresponding to the most number of detected NOEs (Figure 3D, bottom) along the sequence (E5-L7, R12-A16) overlap with the two regions exhibiting the largest alpha-helical propensity of the micelle-bound peptide.

Calculated Solution NMR Structure of the Signal Peptide. The results of structure calculation are summarized in Figure 4 and Table 1. Representative structure of the lowest energy structural ensemble was determined by calculating the mean coordinates using a subroutine of ARIA. According to this, in $50 \%$ methanol, a twelve-residue alpha-helix (A3-N14) is followed by a short $3_{10}$ helix (A16-D18) with a nearly perpendicular orientation. Both helices are well defined yielding an average backbone RMSD from the mean for the whole A3-D18 region of $0.41 \pm 0.07 \AA$. Side-chains in the same ordered region give an overall average RMSD from the mean of $1.3 \pm 0.8 \AA$. The N-terminal half of the $\alpha$-helix is stabilized by a hydrophobic cluster formed by the side chains of residues $A 3,14$, L7, and the apolar segment of the side chain of R6 (Figure 4A, top). We note that a slight kink is introduced into the helix between the two serines manifested in $(\Phi, \Psi)$ dihedral angles slightly deviating from the ideal. The distribution of various residue types around the axis of the approximately $18 \AA$ length $\alpha$-helix gives rise to a strong amphipathic character (Figure 4C). Specifically, side chains of residues A3, 14, L7, and L11 form a hydrophobic patch on one side of the helix ( $\sim 100^{\circ}$ if viewed on the cross-section of the helix), whereas polar residues including the charged ones form an extensive hydrophilic surface. As the charged residues (E5, R6, R12) are distributed throughout the sequence as well, the peptide is endorsed with the capability of engaging in extensive electrostatic interactions both about and along the helical axis. 
Comprised of the short A16-K17-D18 segment, the $3_{10}$-helix has a zwitterionic character. Intriguingly, this is the region, in which peak doubling was observed in $50 \%$ methanol. This may be an indication that the break between the two helices introduces a flexibility into the chain allowing the two helices to uncouple from each other and change their relative orientation. Such a fluctuation may have a functional role for engaging in specific interactions. Additionally, the hinge can modulate the amphipathicity of the peptide chain by modifying the segregation between polar and apolar segments of the surface.

The structure of the micelle-bound peptide while appears to be rather compact, possesses less secondary structure content. The most representative element of the lowest energy structural ensemble exhibits two short $\alpha$-helices in regions A3-L7 and A16-D19 (Figure 4B). Both helices are well defined with average backbone RMSD-s from the mean of $0.53 \pm 0.27 \AA$ and $0.63 \pm 0.23 \AA$, respectively. The two helices are kept proximate to each other by a nine-residue linker engaged in electrostatic interactions with $\alpha-$-II. Specifically, the $N \varepsilon$ atom of R12 is positioned favorably to form a hydrogen bond with the $0 \varepsilon 2$ atom of D18. The position of the latter is further stabilized by an intrahelical salt bridge with the neighboring side chain of K17. The $\mathrm{N}$-terminal $\alpha$-helix of the micelle-bound signal peptide corresponds to the first five residues of the $\alpha$-helix formed in $50 \%$ methanol. This is the segment stabilized by extensive van der Waals interactions in methanol, which although appear to be less tight for the micelle-bound peptide likely provide a substantial source of stabilization for $\alpha-I$ in SDS as well. Similar to our findings in the presence of methanol, the short $\alpha$-helical segment possesses a significant amphipathic character exhibiting a hydrophopic patch comprised of the side chains of $A 3,14$, and L7 (Figure 4D). 


\section{Discussion}

The flagellar export machinery is a specialized type III secretion system which mediates secretion of external flagellar proteins. Self-assembling ability of flagellar axial proteins is controlled by their disordered terminal regions $[1,25]$. Although it seems that the flagellar export signal is located within the $\mathrm{N}$-terminal disordered part of the export substrates, these sequences are highly diverse and share virtually no homology. It was demonstrated that the 26-47 segment of Salmonella flagellin contains the flagellar export signal [11]. The major part of this segment (residues 32-44) forms the irregular extended spoke region when stabilized upon filament formation [19]. In this work we have investigated the conformational properties of the FliC export signal sequence under various conditions.

According to our $\mathrm{H}^{1}$-NMR measurements, in aqueous solution the signal sequence was highly flexible lacking a well-defined structure. However, it became stabilized and assumed a folded structure in solvents mimicking amphipathic environments. Mostly the $\mathrm{N}$-terminal half of the peptide chain showed a significant propensity to form an $\alpha$-helical structure with an amphipathic character. In addition, a short segment in the C-terminal region (A16-D18) also preferentially adopted a helical conformation. It remains elusive how the flagellar export signal is recognized by the flagellar export machinery. The membrane-associated FlhA-FlhB platform is supposed to be responsible for substrate recognition [2], but the molecular details of this process are not known. We believe that conformational properties of the signal sequence carry the clue for understanding the recognition mechanism.

$\mathrm{N}$-terminal targeting signals (TSs) are typical in eukaryotes for protein import into different organelles, including mitochondria, chloroplasts, peroxisomes or the endoplasmic reticulum [26]. Although the particular import mechanisms are remarkably different, the TSs appear to have similar conformational characteristics. Typically, they are structurally disordered but all involve a sequence element with a high propensity to form an $\alpha$-helical segment in which hydrophobic residues cover one side of the helix. Upon interaction with membrane-bound receptor proteins these targeting 
signals radically change their conformation adopting an amphipathic $\alpha$-helical structure which is recognized by the receptor $[27,28]$.

Our results reveal that the flagellar export signal of Salmonella flagellin is capable of acquiring a partially $\alpha$-helical amphipathic structure under appropriate conditions. This observation raises the possibility that amphipathic helical ordering may play an important role in recognition of the export substrates by the flagellum-specific export machinery, resembling the mechanism observed in the case of protein translocation into intracellular organelles.

\section{Acknowledgements}

We thank Dr. Zoltán Bánóczi for peptide synthesis. We are grateful to the Japan Science and Technology Corporation for generous donation of equipment. This work was supported by the Hungarian Scientific Research Fund (OTKA) grants K104726 and K109035. 


\section{References}

1. F. Vonderviszt, K. Namba, Structure, function and assembly of flagellar axial proteins, in: T. Scheibel (Ed), Fibrous Proteins, Landes Biosciences, Austin, 2008, pp. 58-76.

2. T. Minamino, Protein export through the bacterial type III export pathway, Biochim. Biophys. Acta 1843 (2014) 1642-1648.

3. A. Blocker, K. Komoriya, S-I. Aizawa, Type III secretion systems and bacterial flagella: insights into their function from structural similarities, Proc. Natl. Acad. Sci. USA 100 (2003) 3027-3030.

4. A. Diepold, J.P. Armitage, Type III secretion systems: the bacterial flagellum and the injectisome, Philos. Trans. R. Soc. Lond. B Biol. Sci. 370 (2015) 20150020.

5. L. Journet, K.T. Hughes, G.R. Cornelis, Type III secretion: a secretory pathway serving both motility and virulence, Mol. Membr. Biol. 22 (2006) 41-50.

6. T. Minamino, Y.V. Morimoto, N. Hara, K. Namba, An energy transduction mechanism used in bacterial flagellar type III protein export, Nat. Commun. 2 (2011) 475.

7. K. Paul, M. Erhardt, T. Hirano, D.F. Blair, K.T. Hughes, Energy source of flagellar type III secretion, Nature 451 (2008) 489-492.

8. T. Hirano, T. Minamino, K. Namba, R.M. Macnab, Substrate specificity classes and the recognition signal for Salmonella type III flagellar export, J. Bacteriol. 185 (2003) 2485-2492.

9. J.E. Galán, H. Wolf-Watz, Protein delivery into eukaryotic cells by type III secretion machines, Nature 444 (2006) 567-573.

10. H.M. Singer, M. Erhardt, K.T. Hughes, Comparative analysis of the secretion capability of early and late_flagellar_type III secretion substrates, Mol. Microbiol. 93 (2014) 505-520.

11. B.M. Végh, P. Gál, J. Dobó, P. Závodszky, F. Vonderviszt, Localization of the flagellum-specific secretion signal in Salmonella flagellin, Biochem. Biophys. Res. Commun. 345 (2006) 93-98.

12. J. Dobó, J. Varga, R. Sajó, B.M. Végh, P., Gál, P., Závodszky, F. Vonderviszt, Application of a short, disordered $\mathrm{N}$-terminal flagellin segment, a fully functional flagellar type III export signal, to expression of secreted proteins, Appl. Env. Microbiol. 76 (2010) 891-899.

13. M.G. Kornacker, A. Newton, Information essential for cell-cycle-dependent secretion of the 591residue Caulobacter hook protein is confined to a 21-amino-acid sequence near the $\mathrm{N}$-terminus, Mol. Microbiol. 14 (1994) 73-85.

14. C. Weber-Sparenberg, P. Pöplau, H. Brookman, M. Rochón, C. Möckel, M. Nietschke, H. Jung, Characterization of the type III export signal of the flagellar hook scaffolding protein FlgD of Escherichia coli, Arch. Microbiol. 186 (2006) 307-316.

15. F. Vonderviszt, S. Kanto, S.-I. Aizawa, K. Namba, Terminal regions of flagellin are disordered in solution, J. Mol. Biol. 209 (1989) 127-133. 
16. F. Vonderviszt, R. Ishima, K. Akasaka, S-I. Aizawa, Terminal disorder: a common structural feature of the axial proteins of bacterial flagellum, J. Mol. Biol. 226 (1992) 575-579.

17. A. Chacinska, C.M. Koehler, D. Milenkovic, T. Lithgow, N. Pfanner, Importing mitochondrial proteins: machineries and mechanisms, Cell 138 (2009) 628-644.

18. G. Schatz, The protein import machinery of mitochondria. Protein Sci. 2 (1993) 141-146.

19. K. Yonekura, S. Maki-Yonekura, K. Namba, Complete atomic model of the bacterial flagellar filament by electron cryomicroscopy, Nature 424 (2003) 643-650.

20. O. Toke, Z. Bánóczi, P. Király, R. Heinzmann, J. Bürck, A.S. Ulrich, F. Hudecz, A kinked antimicrobial peptide from Bombina maxima. I. Three-dimensional structure determined by NMR in membrane-mimicking environments, Eur. Biophys. J. 40 (2011) 447-462.

21. G. Wagner, A. Pardi, K. Wüthrich, Hydrogen bond length and $1 \mathrm{H}$ NMR chemical shifts in proteins, J. Am. Chem. Soc. 105 (1983) 5948-5949.

22. N.E. Zhou, B-Y. Zhou, B.D. Sykes, R.S. Hodges, Relationship between amide proton chemical shifts and hydrogen bonding in amphipathic $\alpha$-helical peptides, J. Am. Chem. Soc. 114 (1992) $4320-4326$.

23. I.D. Kuntz, P.A. Kosen, E.C. Craig, Amide chemical shifts in many helices in peptides and proteins are periodic, J. Am. Chem. Soc. 113 (1991) 1406-1408.

24. K. Wüthrich, NMR of proteins and nucleic acids, Wiley, New York, 1986.

25. K. Namba, Roles of partly unfolded conformations in macromolecular self-assembly, Genes Cells 6 (2001) 1-12.

26. M. Kunze, J. Berger, The similarity between $\mathrm{N}$-terminal targeting signals for protein import into different organelles and its evolutionary relevance, Front. Physiol. 6 (2015) 259.

27. C.Y. Janda, J. Li, C. Oubridge, H. Hernández, C.V. Robinson, K. Nagai, Recognition of a signal peptide by the signal peptide recognition particle, Nature 465 (2010) 1489-1498.

28. Y. Abe, T. Shodai, T. Muto, K. Mihara, H. Torii, S. Nishikawa, T. Endo, D. Kohda, Structural basis of presequence recognition by the mitochondrial protein import receptor Tom20, Cell 100 (2000) 551-560. 


\section{Figure Captions}

Figure 1 Amide proton regions of one-dimensional ${ }^{1} \mathrm{H}$ spectra of the export signal in (a) $20 \mathrm{mM}$ potassium phosphate, $50 \mathrm{mM}$ potassium chloride, $0.05 \% \mathrm{NaN}_{3}$ at $\mathrm{pH}=6.3$ and $25{ }^{\circ} \mathrm{C}$, (b) $\mathrm{CD}_{3} \mathrm{OH} / \mathrm{H}_{2} \mathrm{O}$ $(1: 1, v / v)$ at $25{ }^{\circ} \mathrm{C}$, and (c) $200 \mathrm{mM} \mathrm{d} 25$ SDS, $10 \mathrm{mM}$ sodium phosphate at $\mathrm{pH}=5.8$ and $40{ }^{\circ} \mathrm{C}$. Resonances for which chemical shift changes are most easily seen are labeled with the corresponding residue number.

Figure 2 Regions of two-dimensional ${ }^{1} \mathrm{H}-{ }^{1} \mathrm{H}$ spectra of the export signal showing the intra- and interresidue correlations used to help establish sequence-specific resonance assignments. Superimposed $\mathrm{H}_{\alpha}-\mathrm{H}_{\mathrm{N}}$ region of 2D TOCSY (black) and 2D NOESY (grey) spectra in (a) $\mathrm{CD}_{3} \mathrm{OH} / \mathrm{H}_{2} \mathrm{O}(1: 1, \mathrm{v} / \mathrm{v}), 25^{\circ} \mathrm{C}$, and (b) SDS micelles $\left(\mathrm{pH}=5.8,40{ }^{\circ} \mathrm{C}\right)$. Alpha-protons are labeled according to their assignment. Resonances showing peak doubling in the presence of methanol are labeled in magenta.

Figure 3 Summary of NMR structural parameters. (a) Deviations of $\mathrm{H}_{\alpha}$ chemical shifts from random coil values for the export signal in 50\% methanol (white), 200 mM SDS (grey), and aqueous buffer (blue line). (b) Deviations of $\mathrm{H}_{\mathrm{N}}$ chemical shifts from random coil values for the export signal in $50 \%$ methanol (•) and in $200 \mathrm{mM}$ SDS (o). (c-d) Diagnostic inter-atomic distance restraints and histograms showing the number of intra- and inter-residue NOEs as a function of the residue number obtained for the export signal in (c) $50 \%$ methanol $\left(25^{\circ} \mathrm{C}\right)$ and (d) $\operatorname{SDS}\left(\mathrm{pH}=5.8,40^{\circ} \mathrm{C}\right)$.

Figure 4 Results of NMR structure calculation. (a-b) Ribbon diagrams of the most representative element of the ten lowest energy structures in (a) 50\% methanol, and (b) SDS micelles. Average backbone RMSD-s from the mean are shown at the bottom and are mapped onto the ribbon diagram in a blue-to-red gradient. Residues with backbone RMSD-s outside of the indicated range are depicted in grey. Van der Waals and electrostatic interactions providing an additional source of stabilization for the secondary structure elements are indicated at the top. (c-d) The same ribbon diagrams as in (a) and (b) showing the amino acid side-chains for the $\mathrm{N}$-terminal $\alpha$-helix. Color coding: hydrophobic residues in grey, positively charged residues in blue, negatively charged residues in red, other polar residues in green, glycines in yellow. 
Table 1. Statistics of the NMR structure and stereochemical quality of the export signal in $\mathrm{H}_{2} \mathrm{O} / \mathrm{CD}_{3} \mathrm{OH}(1: 1, \mathrm{v} / \mathrm{v})\left(25^{\circ} \mathrm{C}\right)$ and SDS micelles $\left(40^{\circ} \mathrm{C}, \mathrm{pH}=5.8\right)$. All statistics were carried out using PROCHECK. The two end-residues and the glycines were excluded from the Ramachandran analysis.

Ensemble RMSD values

$\mathrm{CD}_{3} \mathrm{OH}$

SDS

Average main-chain RMSD from mean coordinates, $\AA$

$\alpha-I$ (A3-N14 in $\mathrm{CD}_{3} \mathrm{OH}, \mathrm{A3}-\mathrm{L} 7$ in SDS)
$3_{10}$ (A16-D18 in $\left.\mathrm{CD}_{3} \mathrm{OH}\right)$
$\alpha-I I$ (A16-D19 in SDS)
overall for A3-D19

$\begin{array}{cc}0.42 \pm 0.08 & 0.53 \pm 0.27 \\ 0.39 \pm 0.03 & - \\ - & 0.63 \pm 0.23 \\ 0.41 \pm 0.07 & 1.3 \pm 1.1\end{array}$

Statistics

Ramachandran plot statistics

Residues in most favored regions $[A, B, L], \%(\#)$

89.5 (17)

$73.7(14)$

Residues in additionally allowed regions $[a, b, l, p], \%$ (\#)

$10.5(2)$

$21.1(4)$

Residues in generously allowed regions [ a, b, I, p ], \% (\#)

$0.0(0)$

$5.3(1)$

Residues in disallowed regions, \% (\#)

$0.0(0)$

$0.0(0)$

Main-chain statistics

SD of $\omega$ angle, degrees

6.9

5.1

Bad contacts $/ 100$ residues

0

$\mathrm{C}_{\alpha}$ chirality, SD of $\zeta$ angle, degrees

0.7

0

$\mathrm{H}$-bond energy, $\mathrm{kcal} / \mathrm{mole}$

1.1

1.1

Overall G-factor

$-0.1$

0.9

$-0.1$

Side-chain statistics

$\chi-1$ gauche minus SD, degrees

25.4

10.1

$\chi-1$ trans SD, degrees

7.4

8.4

$\chi-1$ gauche plus $S D$, degrees

14.3

23.0

$\chi-1$ pooled SD, degrees

16.8

18.8

$\chi-2$ trans SD, degrees

5.5

25.3 


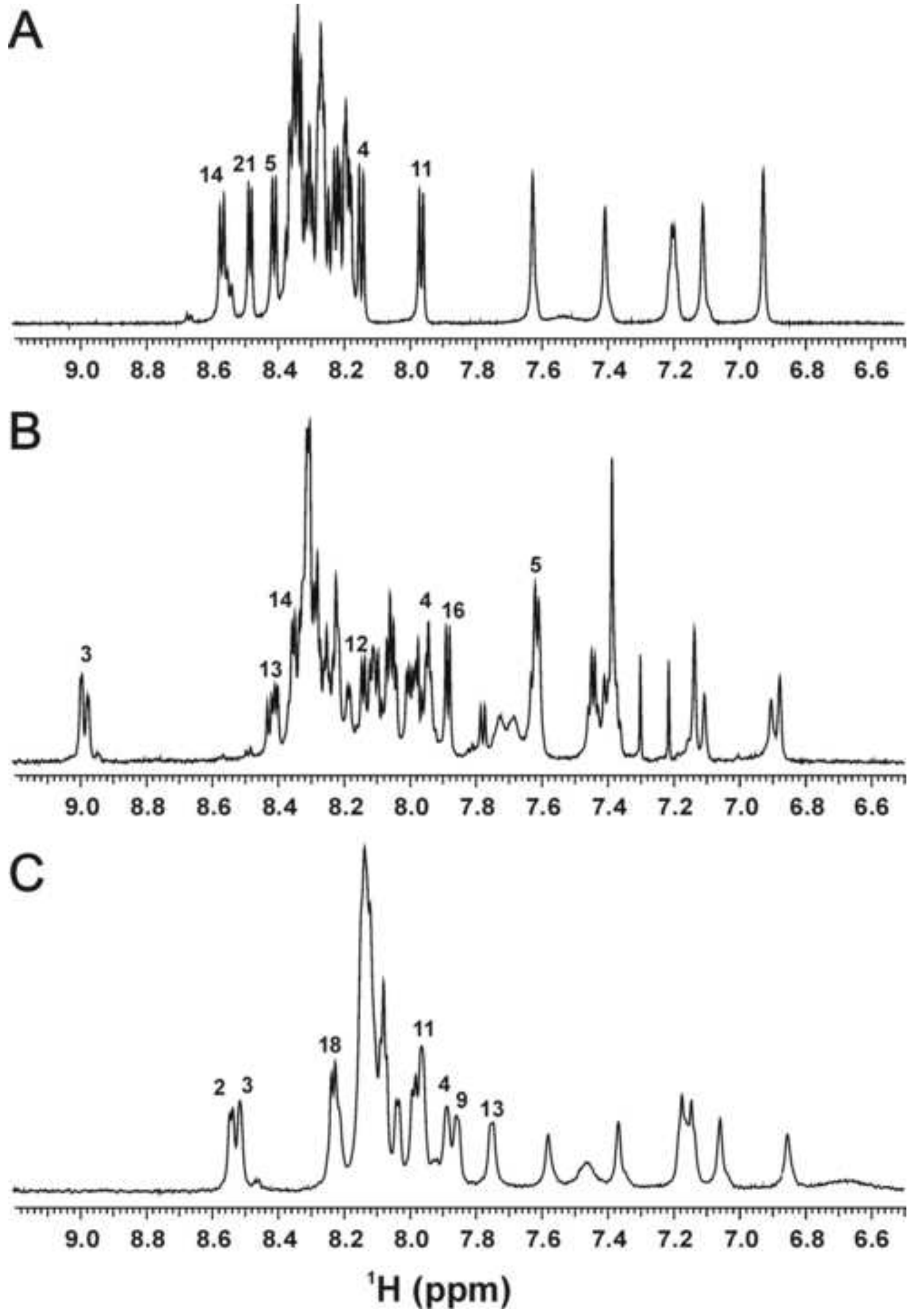



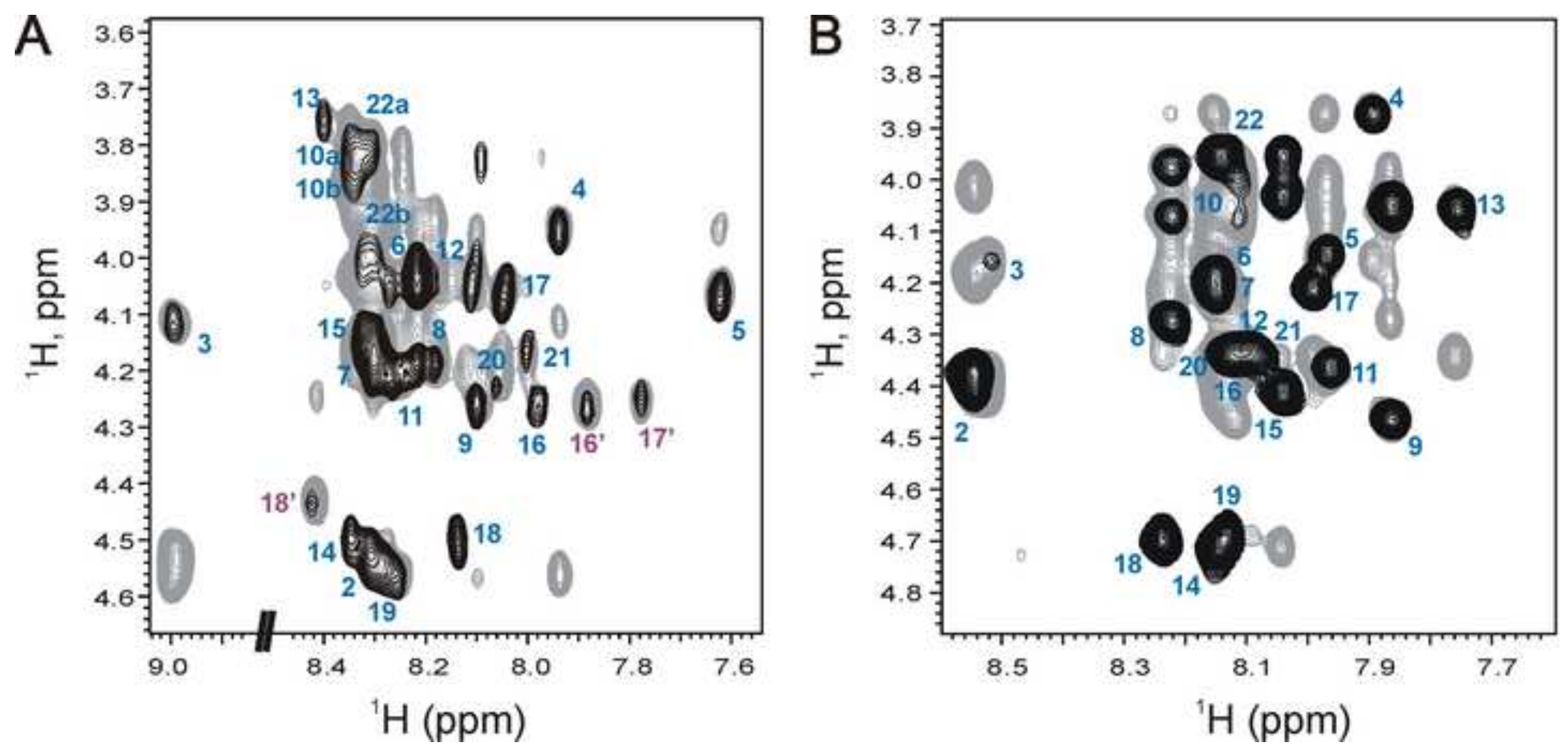
A

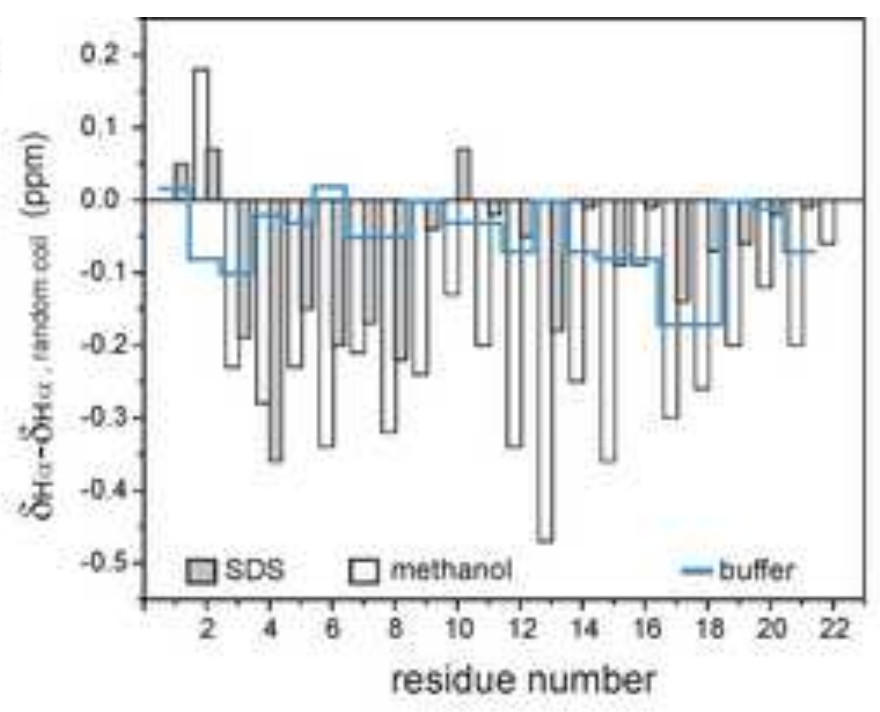

C
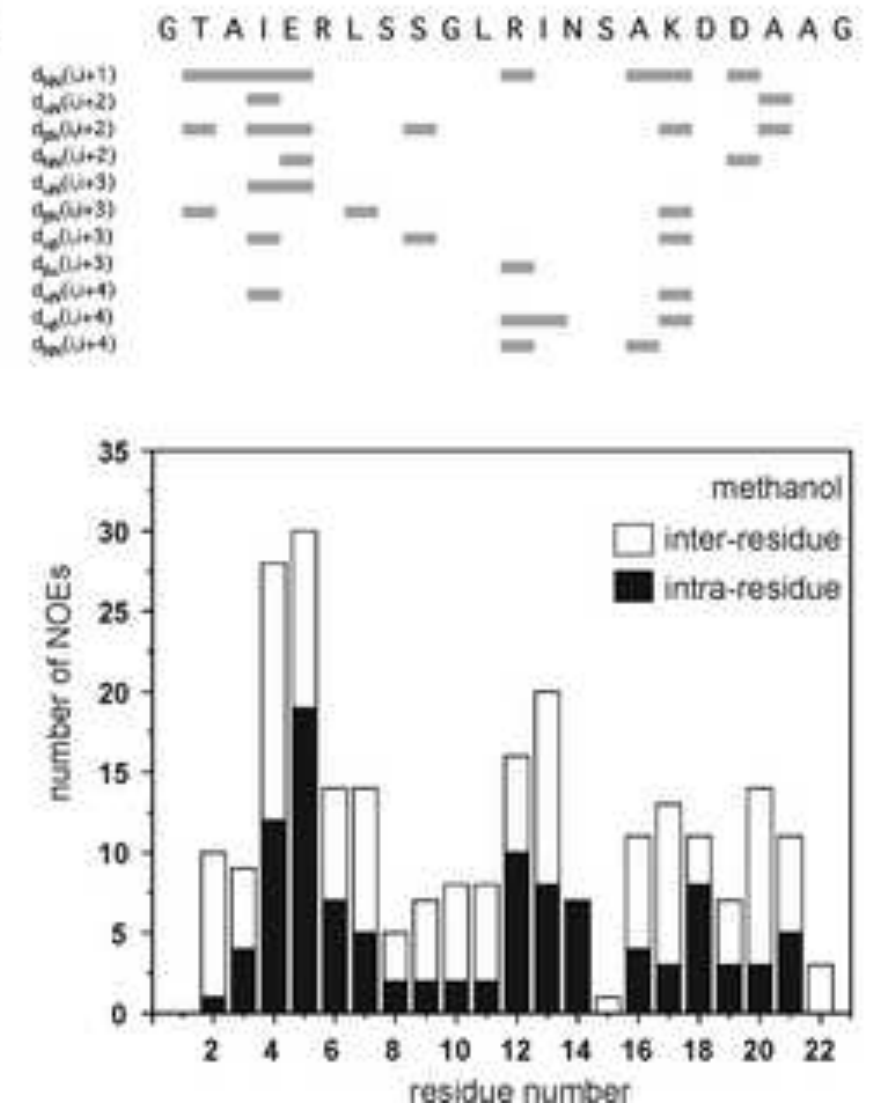

B

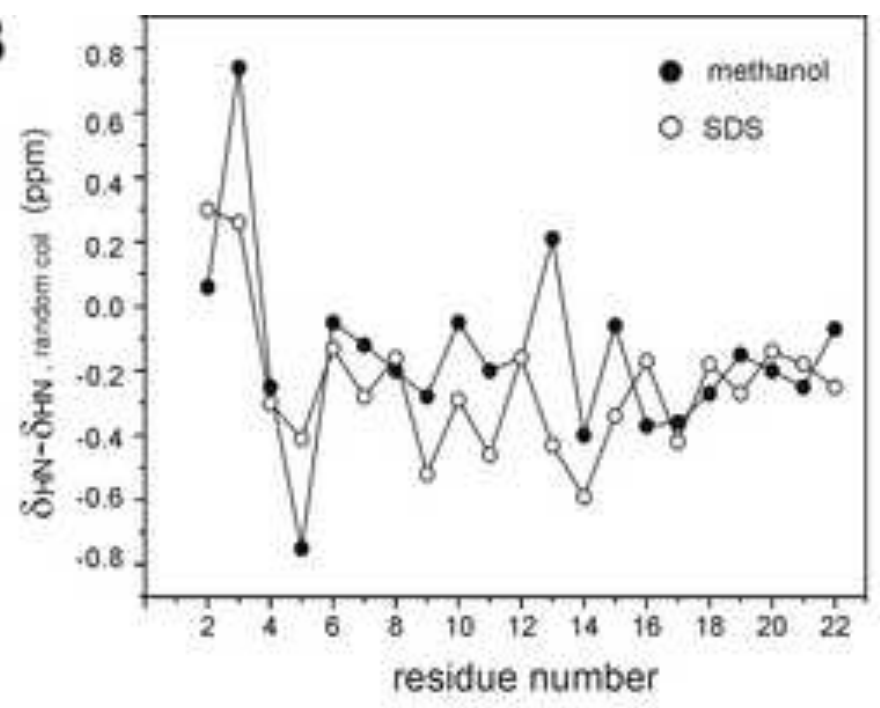

D

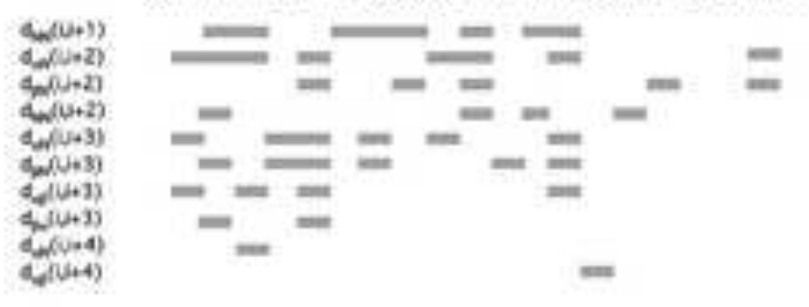

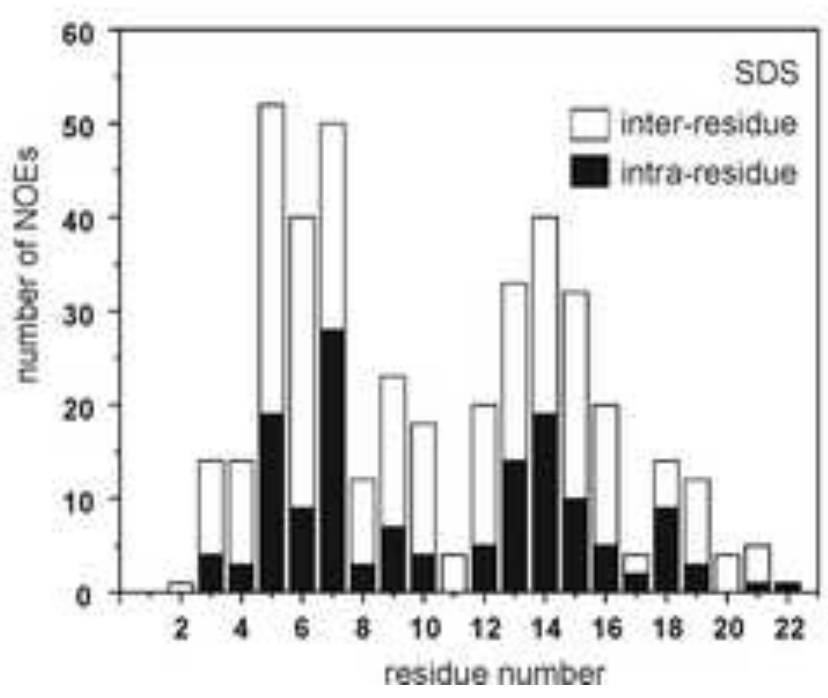


A
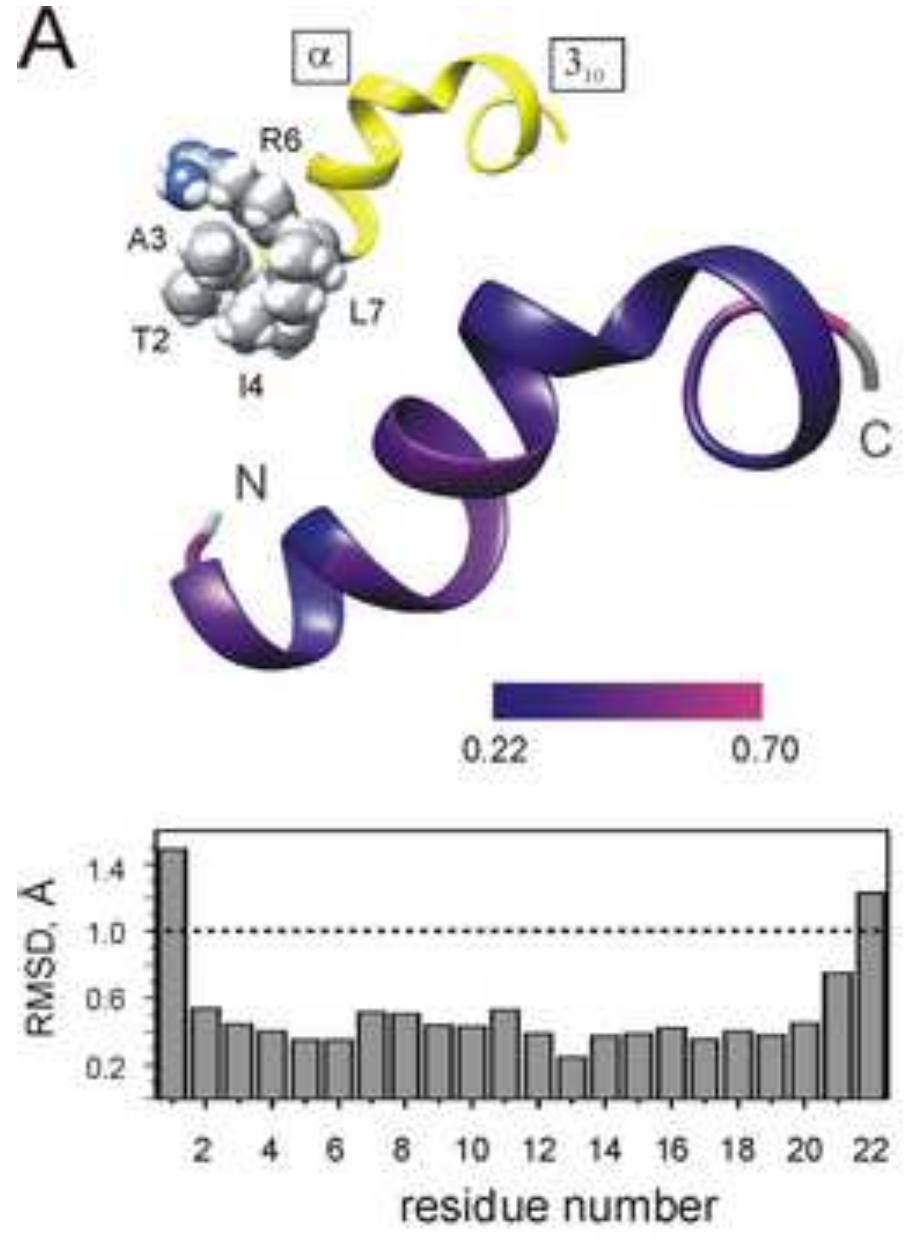

C

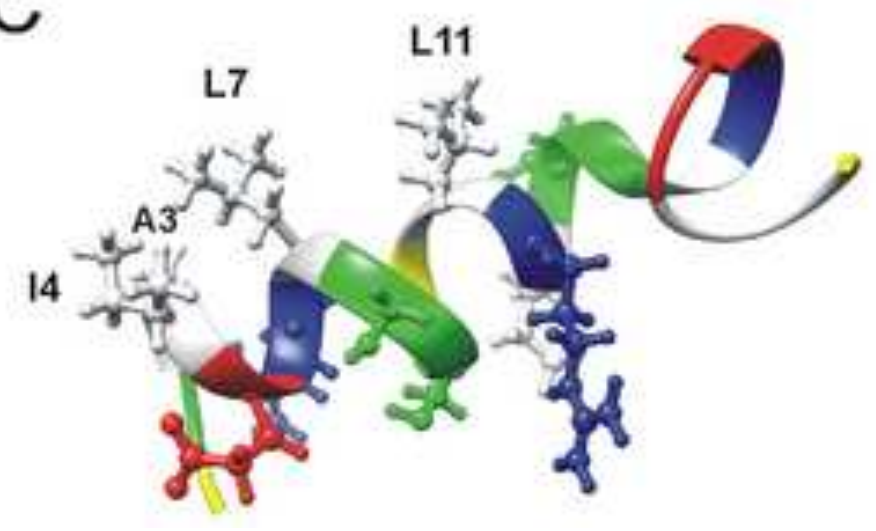

B
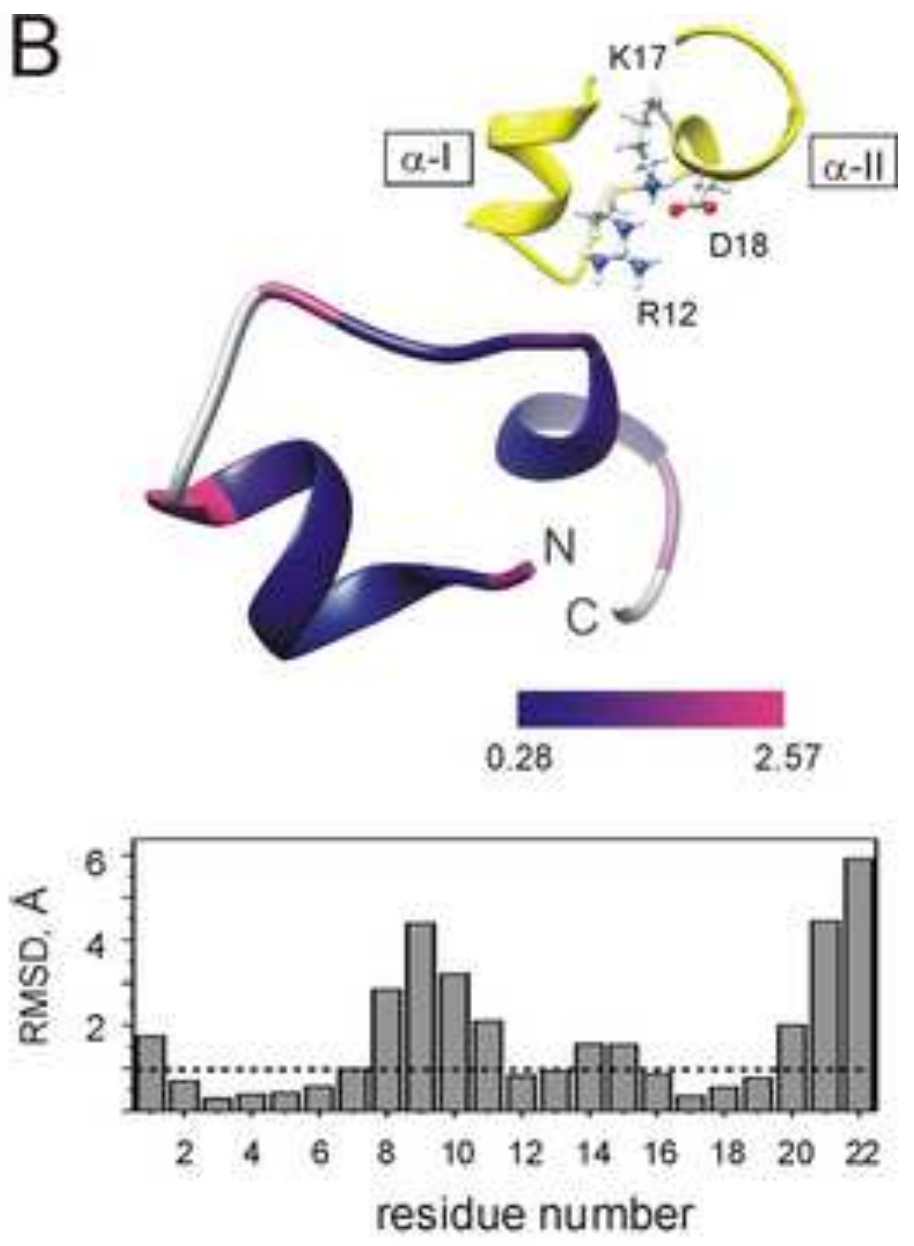

D

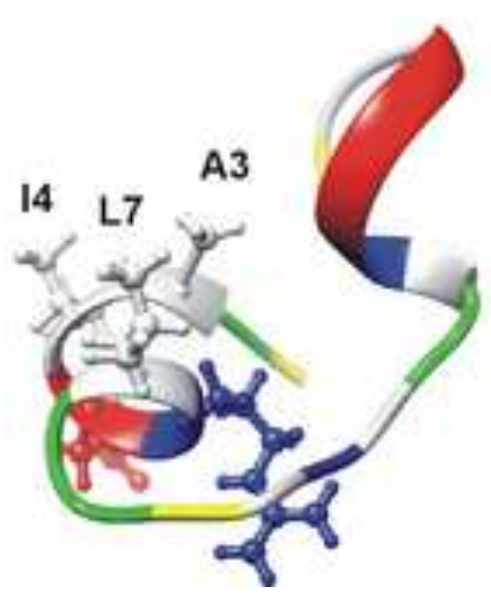




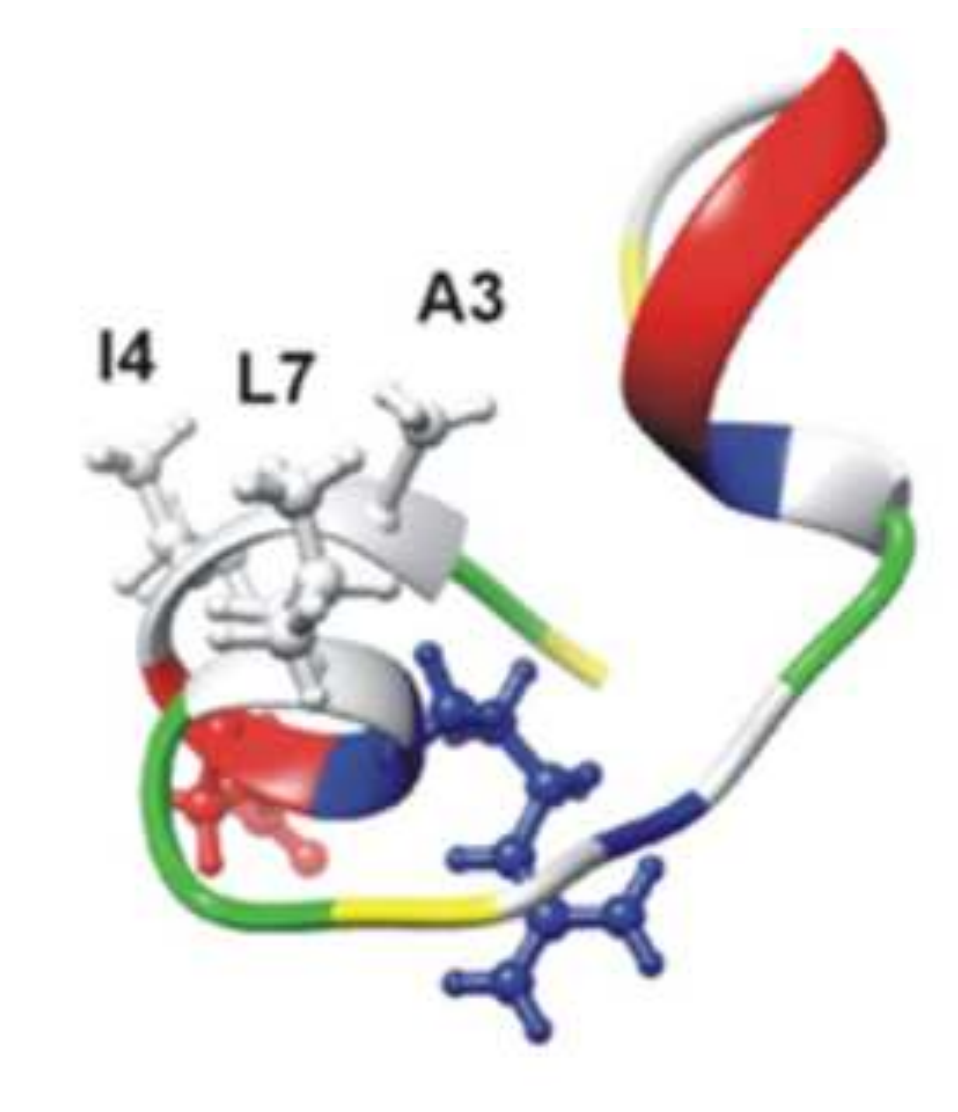

SDS micelles

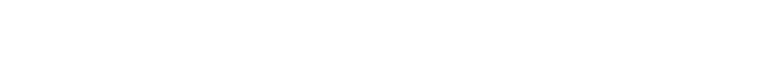

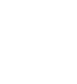

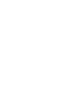
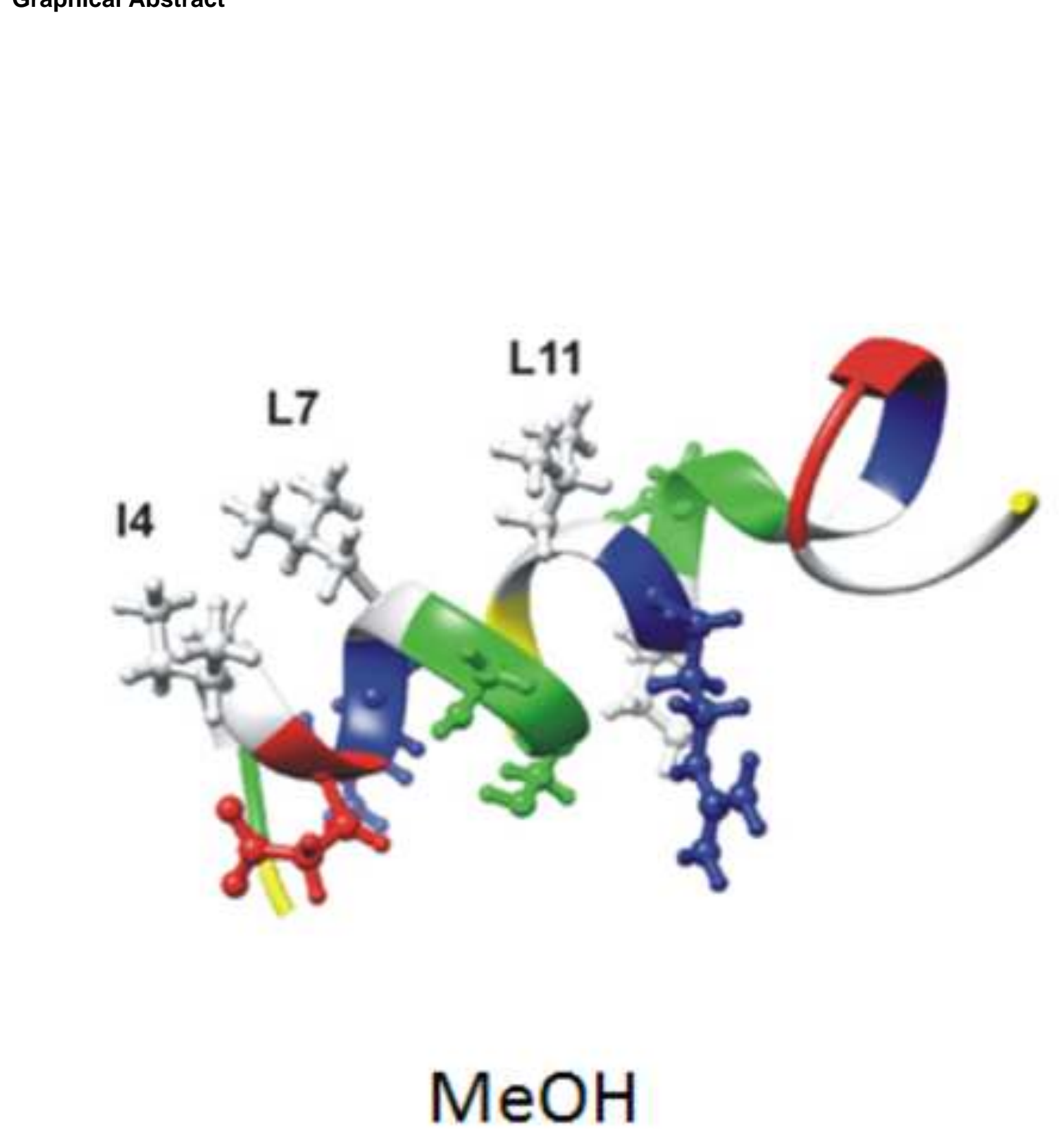

\section{$\mathrm{MeOH}$ \\ $\mathrm{MeOH}$}

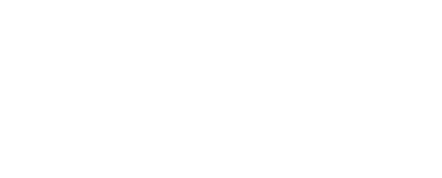

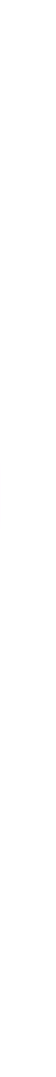
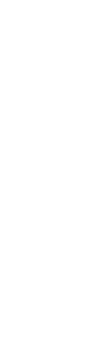